\title{
Preparing for COVID-19: The lessons from SARS 2003 in Canada
}

\author{
David White ${ }^{1}$
}

\author{
${ }^{1}$ Professor \& Vice Chair, Family Doctor Leadership \\ Family \& Community Medicine, University of Toronto \\ Community Teacher, North York General Hospital
}

\author{
Corresponding Author \\ David White: Family \& Community Medicine, University of Toronto \\ E-mail: david.white@utoronto.ca
}

\section{What is Covid-19?}

Definition: COVID-19 is the infectious disease caused by the most recently discovered coronavirus. It was first recognized in Wuhan, China, in December 2019.

Symptoms: The most common symptoms are fever, tiredness, and dry cough. Some people may have aches and pains, nasal congestion, runny nose, sore throat or diarrhea.

Course: Symptoms usually begin gradually. Some people become infected but don't develop any symptoms. Most people (about 80\%) recover from the disease without needing special treatment. Around 1 out of every 6 people becomes seriously ill and develops difficulty breathing.

At risk: Older people, and those with underlying medical problems. People with fever, cough and difficulty breathing should seek medical attention.

\section{How to prevent it}

Currently there is no immunization and no specific treatment for COVID-19. The virus spreads by droplets travelling from one person to another, due to coughing or coming into contact with body fluids. Prevention relies on limiting transmission of the virus among populations, between individuals and in health care settings. Population controls include "social distancing" such as cancelling large gatherings and limiting travel. Individual measures include regular handwashing, covering your cough, staying 1 meter away from someone who is coughing. The WHO's full list of prevention recommendations is on their Q\&A site: https://www.who.int/news-room/q-a-detail/qa-coronaviruses. Measures for health care settings are discussed in the next section.

\section{How to prepare Family Doctors and frontline providers: lessons from SARS}

Toronto, Canada faced the most severe outbreak of SARS outside of China, Hong Kong and Taiwan. The only physician to die of SARS was a family doctor. Here are the lessons we learned that can help prepare for other pandemics, with a focus on primary care.

1. System planning: Pandemics can only be managed if all involved sectors contribute to planning. Within health care, the perspectives and expertise of family doctors and other primary care providers must inform the plan, along with experts in public health, infectious disease, hospital care and emergency preparedness.
2. Coordinated, effective communication: Regular communication from a reliable, trusted source is essential. It needs to be appropriate for the specific audience, such as the general public, all health care workers, or a specific sector. Messages for different settings inevitably will vary, but should be coordinated so as not to conflict, and the rationale for differences should be clear.

Communication needs to be two-way, and is effective only if received and acted upon. Ensure that health care workers can easily receive or access the information they need, and can incorporate new practices into their daily routines. Frontline health care workers are the "eyes and ears" in managing a pandemic. They need to be able to channel their observations, perspectives and questions to those who are in charge, and have these incorporated into the response to the pandemic.

3. Regular updates: Pandemic management constantly evolves as new information becomes available. We know a lot more about COVID-19 in a much shorter time than we did about SARS. Nevertheless, our understanding changes as we learn more about the virus, its spread and its management. Clear and timely updates build confidence. Suppressing information or failing to acknowledge that previous advice is out-ofdate (or simply wrong) leads to confusion, distrust, and fear.

4. Build capacity: In general, health care systems operate at $100 \%$ capacity all the time. Hospitals are usually full, emergency departments are crowded, family doctors are busy. The challenge is to reconfigure services to cope with an escalation in demand, while preserving and protecting core functions. Current evidence shows that most people who contract COVID-19 recover, while a small percentage become seriously ill and require hospital care.

5. Protect health care workers: Those providing health care need to be safe at work. SARS took a terrible toll on doctors and nurses, and seriously affected the operations of all the hospitals in Toronto. Everyone in health care needs to have appropriate personal protective equipment (PPE) - this requires supply management. They must be able to use it properly when providing care to people with any respiratory infection.

6. Triage: Patients with respiratory illnesses need to be managed in a way that provides the right care in the right location and minimizes spread. Most people do 
not need to see a doctor. Messages to the population at large should emphasize self-monitoring, self-isolation if ill, and when to seek attention. Ideally, anyone with a new respiratory illness who thinks they need assessment should call their health care provider first for advice. Most can manage at home. Those with severe illness and shortness of breath should be directed to an emergency department, and travel in a way to minimize the risk of infection to others. An intermediate group will require assessment in a safe primary care setting. During SARS in Toronto we set up special assessment centres, with the right equipment and trained personnel. Current plans are to do the same for COVID-19. Doctors and nurses will volunteer to work there in shifts. This allows most family physicians' offices to continue to provide regular care in a safe manner, and takes pressure of hospital emergency departments.

7. Overload, stress and fear: An esteemed infectious disease expert remarked in a forum for family doctors, "Managing a pandemic is a marathon, not a sprint." A vivid lesson from SARS is that those working at the centre of the outbreak were overloaded, caring for co-workers who had become desperately ill, while suppressing fears that they, too, would fall victim. Entire populations can become panicked, especially if information is withheld or distorted, supplies run out, or services are overwhelmed. Family doctors, trusted by their patients and the public at large, can work together to create a sustainable, robust response.

\section{Closing thoughts}

I am a family doctor in Toronto, Canada, with direct experience with SARS and major involvement in creating the Ontario Health Plan for an Influenza Pandemic. I know very little about Asia Pacific region and its health care system. Nevertheless, I believe Asia Pacific has some major advantages in facing COVID-19. As of this writing, on March $8^{\text {th }} 2020$, the WHO reports 7.134 cases in Republic of Korea, South Korea, 138 cases in Singapore, 455 cases in
Japan, 93 cases in Malaysia, 50 cases in Thailand, 4 cases of COVID-19 in Indonesia, 74 cases in Australia, and 5 cases in New Zealand. Compared with the live-data from https://www.worldometers.info/coronavirus/countrieswhere-coronavirus-has-spread/ on March $09^{\text {th }} 2020$ there were 7.513 cases in Republic of Korea, South Korea, 160 cases in Singapore, 530 cases in Japan, 117 cases in Malaysia, 50 cases in Thailand, 19 cases of COVID-19 in Indonesia, 93 cases in Australia, and 5 cases in New Zealand. The data of patients with COVID-19 show a much lower death rate in those under 50, and no fatalities in children under 10. Appropriate travel restrictions could allow many parts of the region to avoid COVID-19 entirely. Countries with universal health care have a huge advantage, because everyone can have access to the care they need. I hope that sharing my experience will help.

\section{REFERENCES}

1. Information in this document is adapted from the WHO coronavirus website: https://www.who.int/emergencies/diseases/novelcoronavirus-2019

2. As an example, see the attachment, extracted from Ontario Agency for Health Protection and Promotion, Provincial Infectious Diseases Advisory Committee. Routine Practices and Additional Precautions in All Health Care Settings. 3rd edition. Toronto, ON: Queen's Printer for Ontario; November 2012.

3. See: http://www.health.gov.on.ca/en/pro/programs/emb/pan_flu/ pan_flu_plan.aspx 